\title{
The NMSSM dark sector constraints
}

\author{
Albina V. Gurskaya ${ }^{1, \star}$ and Mikhail V. Dolgopolov ${ }^{1, \star \star}$ \\ ${ }^{1}$ Samara University, Academician Pavlov 1, 443011 Samara, Russia
}

\begin{abstract}
In this paper authors present review of neutralino research results in LHC, cosmological observations and theoretical models. Nowdays there are constraints on the mass of dark matter particle from experiments. The neutralino as a candidate for the dark matter particle in the framework of supersymmetry is considered. There are some discuss advantages for it about the NMSSM model. The properties of neutralino are functions of free parameters of the model. In agreement with the foregoing, constraints on free parameters of the NMSSN are determined.
\end{abstract}

\section{Introduction}

Despite the fact that the problem of dark matter originates in the astronomical observation [1], this phenomenon can be explained in the framework of quantum field theory. There is the reason to believe that dark matter consists of new hypothetical particles. In particular, we can make this conclusion based on the paper [2] in which the cosmic microwave background (CMB) was investigated. At the time of formation of the $\mathrm{CMB}$, the baryonic matter contribution to the full density of the Universe was one fifth. It means that $80 \%$ [3] was the dark matter at that time. It can be concluded that the phenomenon of dark matter is the cosmological only and a discussion on new particles is also relevant.

The most popular candidate is the supersymmetric neutralino. Supersymmetric models can also differ in composition of fields, and therefore, the neutralino properties may be different in models. The simplest extension of the standard model MSSM [4] has a very convincing basis. MSSM Superpotential contains the so-called $\mu$-term $\left(\mu H_{1} H_{2}\right)$ including dimensional mass parameter of the Higgs field $\mu$, the value of which cannot be accounted for and matched to masses scale of order TeV to ensure an acceptable range of Higgs bosons masses. Within NMSSM [5] $\mu$-term is dynamically generated by spontaneous symmetry breaking $\left(\lambda S\left(H_{1} H_{2}\right)\right.$, where $\langle S\rangle=v_{3} / \sqrt{2}$ and $\left.\mu=\lambda v_{3} / \sqrt{2}\right)$. This is the most reasonable, moreover, due to new Higgs field the model becomes more flexible to build new parametric scenarios.

Neutralino is not the only candidate for the role of dark matter particles. There is also a prediction of the axion in the NMSSM. It is necessary to note that an appearance of the axion can be realize in various symmetries (R-symmetry [6], Peccei-Quinn [7]). We can exclude this particle picking the appropriate components in superpotential Higgs. In particular, the component $\sim \kappa S^{3}$ [5] provides Peccei-Quinn violation.

\footnotetext{
^e-mail: a-gurska@yandex.ru

$\star \star$ e-mail: mvdolg@yandex.ru
} 
Previous papers were devoted to NMSSM with violation of CP invariance in the Higgs sector [8]. In the models describing elementary particles special place is prepared for the Higgs boson. It was shown that the Higgs field strongly associated with the problem of the baryon asymmetry[9].

Additional CP violating phases enrich scenarios for the physical parameters values of the model, however, it can be difficult to coexist in the case of CP-even and CP-odd state Higgs bosons mixing. The problem of the mass calculating in this case leads to the rigidly fine tuning parameters fixing.

\section{Experimental data for dark matter particles}

There is no conclusive evidence of supersymmetric particles up to now. The data of the Large Hadron Collider (LHC) give us constraints for supersymmetric particles masses. The main attention should be paid to quarks superpartners (squarks) and gluons (gluino). Such particles participate in the strong interaction and must be well created in protons collisions. Therefore gluino with the mass of 1-2 TeV can be discovered at the LHC. But here we have constraints above $1 \mathrm{TeV}$ already. The figure 1 illustrate this situation.

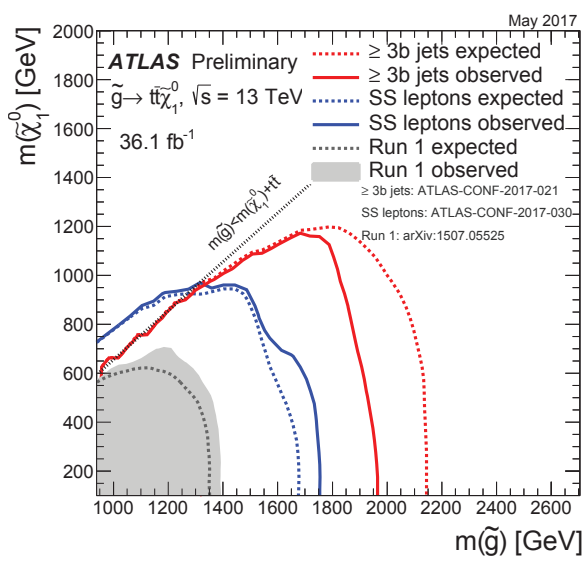

Figure 1. The neutralino mass dependence on the gluino mass in the decay to the top quark pair and an invisible neutralino [10].

The absence of a signal allows to exclude the areas bounded by solid curves. The gray area has been closed according to the results of Run 1 . Along with the considered process also there are many other results.

Different supersymmetric models are checked on the LHC at this moment. For example, figure 2 shows fragment of data set of results of the ATLAS collaboration. There are no signals of neutralino and we have to conclude that constraints for this particle may be above 1-2 TeV and larger. Data which is presented in figures $1 \& 2$ is relevant for May 2017.

But some modified models give different predictions and can allow the light particle. We will discuss this below. In particular, the NMSSM has soft requirements of physical parameters values for neutralino. In addition, for example, the study of Higgs boson invisible decays to neutralino is one of the important and interesting problem in practice and in theory [11].

With regard to direct detection experiments they are designed to detect the nuclear recoil in the scattering of galactic WIMPs on nuclei target. The signal rate depends on the local density and 


\begin{tabular}{|c|c|c|c|c|c|c|c|c|c|}
\hline \multicolumn{2}{|r|}{ Model } & $e, \mu, \tau, \gamma$ & Jets & $E_{T}^{\text {miss }}$ & \multicolumn{2}{|c|}{$\int \mathcal{L} d t\left[\mathrm{fb}^{-1}\right]$} & Mass limit & & \\
\hline \multirow{12}{*}{ 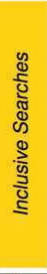 } & MSUGRACMSSM & $0-3 e, \mu / 1-2 \tau$ & $2-10$ jets $/ 3 b$ & Yes & 20.3 & $\frac{\tilde{q}, \tilde{g}}{\tilde{g}}$ & & & $1.85 \mathrm{TeV}$ \\
\hline & $\begin{array}{l}q \tilde{q}, \tilde{q} \rightarrow q x \\
\tilde{q} \tilde{q}, \tilde{q} \rightarrow q \tilde{\chi} \tilde{X}_{1}^{0} \text { (compressed) }\end{array}$ & mono-jet & $1-3$ jets & $\begin{array}{l}\text { Yes } \\
\text { Yes }\end{array}$ & $\begin{array}{r}6.1 \\
3.2\end{array}$ & $\frac{q}{\bar{q}}$ & & $\mathrm{GeV}$ & $1.5 \% \mathrm{leV}$ \\
\hline & $\tilde{g} \tilde{g}, \tilde{g} \rightarrow q \tilde{q} \tilde{\chi} \tilde{\chi}_{1}^{0}$ & 0 & $2-6$ jets & Yes & 36.1 & $\overline{\mathrm{g}}$ & & & $2.02 \mathrm{TeV}$ \\
\hline & $\tilde{g} \tilde{g}, \tilde{g} \rightarrow q q \tilde{\chi}_{1}^{ \pm} \rightarrow q q W^{ \pm} \tilde{\chi}_{1}^{0}$ & 0 & $2-6$ jets & Yes & 36.1 & $\tilde{g}$ & & & $2.01 \mathrm{TeV}$ \\
\hline & $\tilde{g} \tilde{g}, \tilde{g} \rightarrow q q(\ell \ell / v v) \tilde{X}_{1}^{0}$ & $3 e, \mu$ & 4 jets & - & 36.1 & $\bar{g}$ & & & $1.825 \mathrm{TeV}$ \\
\hline & $\tilde{g} \tilde{g}, \tilde{g} \rightarrow q q W Z \tilde{X}_{1}^{0}$ & 0 & $7-11$ jets & Yes & 36.1 & $\bar{g}$ & & & $1.8 \mathrm{TeV}$ \\
\hline & GMSB ( $($ NLSP) & $1-2 \tau+0-1 \ell$ & $\ell \quad 0-2$ jets & Yes & 3.2 & $\tilde{g}$ & & & $2.0 \mathrm{TeV}$ \\
\hline & GGM (bino NLSP) & $2 \gamma$ & - & Yes & 3.2 & $\tilde{\mathrm{g}}$ & & & $1.65 \mathrm{TeV}$ \\
\hline & GGM (higgsino-bino NLSP) & $\gamma$ & $1 b$ & Yes & 20.3 & $\tilde{g}$ & & & $37 \mathrm{TeV}$ \\
\hline & GGM (higgsino-bino NLSP) & $\gamma$ & 2 jets & Yes & 13.3 & $\bar{g}$ & & & $1.8 \mathrm{TeV}$ \\
\hline & GGM (higgsino NLSP) & $2 e, \mu(Z)$ & 2 jets & Yes & 20.3 & $\overline{\mathrm{g}}$ & & $900 \mathrm{GeV}$ & \\
\hline & Gravitino LSP & 0 & mono-jet & Yes & 20.3 & $F^{1 / 2}$ scale & & $865 \mathrm{GeV}$ & \\
\hline \multirow{3}{*}{ 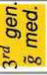 } & $\tilde{g} \tilde{g}, \tilde{g} \rightarrow b \bar{b} \tilde{\chi}_{1}^{0}$ & 0 & $3 b$ & Yes & 36.1 & $\tilde{g}$ & & & $1.92 \mathrm{TeV}$ \\
\hline & $\tilde{g} \tilde{g}, \tilde{g} \rightarrow t \tilde{\hat{\chi}} \tilde{\chi}_{1}^{0}$ & $0-1 e, \mu$ & $3 b$ & Yes & 36.1 & $\tilde{g}$ & & & $1.97 \mathrm{TeV}$ \\
\hline & $\tilde{g} \tilde{g}, \tilde{g} \rightarrow b b \tilde{X}_{1}^{+}$ & $0-1 e, \mu$ & $3 b$ & Yes & 20.1 & $\tilde{g}$ & & & $37 \mathrm{TeV}$ \\
\hline \multirow{8}{*}{ 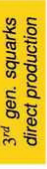 } & $\tilde{b}_{1} \tilde{b}_{1}, \tilde{b}_{1} \rightarrow b \bar{\chi}_{1}^{0}$ & 0 & $2 b$ & Yes & 36.1 & $\tilde{b}_{1}$ & & $950 \mathrm{GeV}$ & \\
\hline & $\tilde{b}_{1} \tilde{b}_{1}, \tilde{b}_{1} \rightarrow \tilde{X}_{1}^{ \pm}$ & $2 e, \mu$ (SS) & $1 b$ & Yes & 36.1 & $\tilde{b}_{1}$ & & $-700 \mathrm{GeV}$ & \\
\hline & $\tilde{t}_{1} \tilde{t}_{1}, \tilde{t}_{1} \rightarrow b \tilde{X}_{1}^{ \pm}$ & $0-2 e, \mu$ & $1-2 b$ & Yes & $4.7 / 13.3$ & $\tilde{t}_{1} \quad 117-170 \mathrm{GeV}$ & & $-720 \mathrm{GeV}$ & \\
\hline & $\tilde{t}_{1} \tilde{t}_{1}, \tilde{t}_{1} \rightarrow W b \tilde{\chi}_{1}^{0}$ or $\tilde{\chi}_{1}^{0}$ & $0-2 e, \mu \quad 0$ & $0-2$ jets $/ 1-2 b$ & $b$ Yes & $20.3 / 36.1$ & $\begin{array}{ll}\tilde{i}_{1} & 90-198 \mathrm{GeV}\end{array}$ & & $205-950 \mathrm{GeV}$ & \\
\hline & $\tilde{t}_{1} \tilde{t}_{1}, \tilde{t}_{1} \rightarrow c \tilde{X}_{1}^{0}$ & 0 & mono-jet & Yes & 3.2 & $\tilde{t}_{1}$ & $90-323 \mathrm{GeV}$ & & \\
\hline & $\tilde{t}_{1} \tilde{t}_{1}$ (natural GMSB) & $2 e, \mu(Z)$ & $1 b$ & Yes & 20.3 & $\tilde{t}_{1}$ & $150-6$ & GeV & \\
\hline & $\tilde{t}_{2} \tilde{t}_{2}, \tilde{t}_{2} \rightarrow \tilde{t}_{1}+Z$ & $3 e, \mu(Z)$ & $1 b$ & Yes & 36.1 & $\tilde{t}_{2}$ & & $290-790 \mathrm{GeV}$ & \\
\hline & $\tilde{t}_{2} \tilde{t}_{2}, \tilde{t}_{2} \rightarrow \tilde{t}_{1}+h$ & $1-2 e, \mu$ & $4 b$ & Yes & 36.1 & $\tilde{t}_{2}$ & & $320-880 \mathrm{GeV}$ & \\
\hline
\end{tabular}

Figure 2. Mass reach of ATLAS searches for Supersymmetry. Fragment of the table [10].

velocity distribution of WIMPs in our Galaxy, the WIMP mass, and the interaction cross-section on the target nuclei [12]. We have no prediction on mass in this case yet, but the experiment gives us constraints on the WIMP-neutron scattering cross-section correlating dark matter particle mass. In the next figure 3 we can see data of 2017 year [13].

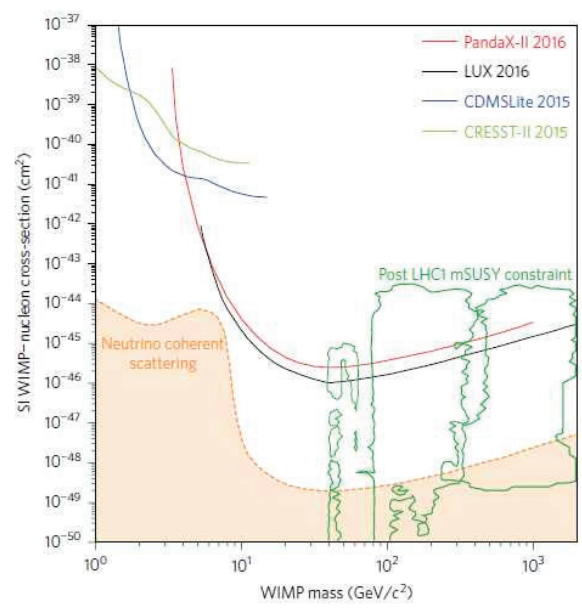

Figure 3. Upper limits on the spin-independent WIMP-nucleon scattering cross-section set by current leading experiments. The limit curves are from PandaX-II [14], LUX[15], SuperCDMS (CDMSLite)[16] and CRESSTII [17].

The sensitivity of detectors for the direct search of dark matter to the mass of the particle and to the cross section can achive the neutrino area. The scattering processes of neutrinos are background and 
represent the difficult in the registration of new particles. There is acceptable area for supersymmetric particles, but even here the neutrino background is the place to be.

There is amount of continuing attempts to observe the variety of products of annihilation or decay of dark matter: positrons, neutrinos, antiprotons, etc. However, there is indirect benefit to search them from photons. Photons are not deflected by magnetic fields and clearly indicate the source. In the paper [18] there is the description of methods for detecting of dark matter annihilations. It should be noted that the cross section of annihilation may not match the sensitivity of the telescopes. However in the past few years gamma-ray observations give some stringent constraints for annihilating dark matter. The lower limits of dark matter mass annihilating via $e^{+} e^{-}, \mu^{+} \mu^{-}, \tau^{+} \tau^{-}, g g, u \bar{u}$ and $b^{+} b^{-}$ channels are $40 \mathrm{GeV}, 28 \mathrm{GeV}, 30 \mathrm{GeV}, 57 \mathrm{GeV}, 58 \mathrm{GeV}$ and $66 \mathrm{GeV}$ [19].

\section{Theoretical aspects of constrains for physical properties of neutralino}

From the theory point of view all physical properties of the models are functions of free parameters, in particular, the physical properties of the neutralino also. In this report we has interest in the NMSSN dark sector.

The definition of the basis in the neutralino sector is following:

$$
\psi^{0}=\left(-i \tilde{b}^{0},-i \tilde{\omega}^{3}, \tilde{h}_{1}^{0}, \tilde{h}_{2}^{0}, \tilde{s}\right)^{\mathrm{T}},
$$

here $\tilde{b}^{0}, \tilde{\omega}^{3}, \tilde{h}_{1}^{0}, \tilde{h}_{2}^{0}, \tilde{s}$ - bino, wino, higgsino and singlino spinors. The mass part of the Lagrangian has the form:

$$
\mathcal{L}_{m}=-\frac{1}{2}\left(\psi^{0}\right)^{\mathrm{T}} Y \psi^{0}+\text { h.c. }
$$

where $Y$ is:

$$
Y=\left(\begin{array}{ccccc}
M_{1} & 0 & -M_{Z} s_{W} c_{\beta} & M_{z} s_{W} s_{\beta} & 0 \\
0 & M_{2} & M_{Z} c_{W} c_{\beta} & -M_{Z} c_{W} s_{\beta} & 0 \\
-M_{Z} s_{W} c_{\beta} & M_{Z} c_{W} c_{\beta} & 0 & -\lambda v_{3} & -\lambda v_{2} \\
M_{Z} s_{W} s_{\beta} & -M_{Z} c_{W} s_{\beta} & -\lambda v_{3} & 0 & -\lambda v_{1} \\
0 & 0 & -\lambda v_{2} & -\lambda v_{1} & 2 \kappa v_{3}
\end{array}\right) .
$$

Here $M_{1}, M_{2}-\mathrm{U}(1)$ and $\mathrm{SU}(2)-$ mass parameters of the soft violations supersymmetry fields $\tilde{b}^{0}$, $\tilde{\omega}^{3}$ accordingly; $s_{W}=\sin \theta_{W}, c_{W}=\cos \theta_{W} ; \theta_{W}$ - the electroweak mixing angle (Weinberg angle); $s_{\beta}=\sin \beta, c_{\beta}=\cos \beta, M_{Z}-$ mass of $Z$-boson.

The following parameters have no fixed values and also they have no predictions from the model and their value is unknown in the experiment [20,21]:

$$
\begin{gathered}
1.0<\operatorname{tg} \beta \leq 60, \quad M_{1}=M_{2}, \quad 100 \mathrm{GeV} \leq M_{2} \leq 2000 \mathrm{GeV} \\
0.0001 \leq \lambda \leq 0.7, \quad 0 \leq \kappa \leq 0.65 \\
0 \mathrm{GeV} \leq A_{\lambda} \leq 1000 \mathrm{GeV}, \quad-100 \mathrm{GeV} \leq A_{\kappa} \leq-10 \mathrm{GeV}
\end{gathered}
$$

These ranges of values were obtained by scanning of free parameters space on the mass superparticles scale. They were used by the authors of the given report to study the neutralino masses in the MSSM and NMSSM in the case of CP-invariance in the Higgs sector [22]. It is interesting to note that the values of the lightest neutralino NMSSM achives to $962.9 \mathrm{GeV}$, no larger, which is irrelevant to experimental limitations at the LHC, however, do not contradict the data of other methods for dark matter particles detection. But this value in the model obtained at fixed free parameters, 
therefore, the key theoretical restriction on the properties of dark matter are the allowing areas for free parameters of the model.

There is the scan of the free parameters in the model given the constraints on the neutralino mass used data of annihilation of dark matter particles (as the lower constrain we choose $28 \mathrm{GeV}$ ) on figure 4 .
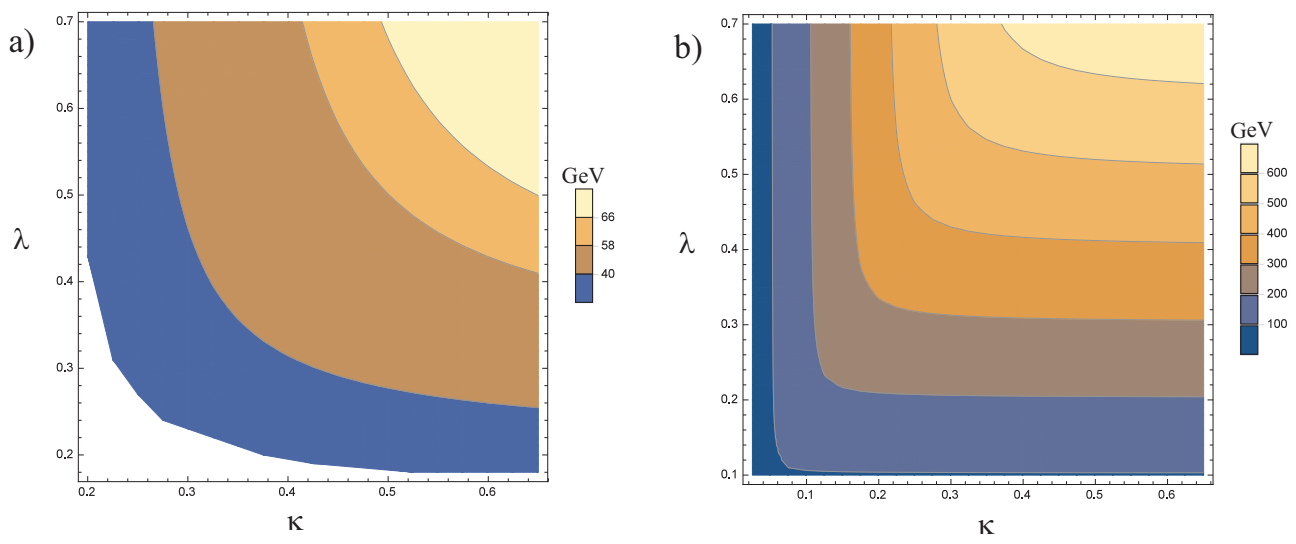

Figure 4. The space of parameters $\lambda$ and $\kappa$ correlating with low neutralino masses constrains. a) Two parameters are fixed: $M_{2}=2000 \mathrm{GeV}$ and $v_{3}=200 \mathrm{GeV}$. The white area corresponds to forbidden values of $\lambda$ and $\kappa$. b) It is the case for $M_{2}=2000 \mathrm{GeV}$ and $v_{3}=1000 \mathrm{GeV}$.

The strong constraint on parameters may be the presence of CP-violating phases in the extended Higgs sector. We can define spontaneous violation of $\mathrm{CP}$-invariance directly in the determination of doublet fields with use of phases $\theta$ and $\varphi$ :

$$
\begin{gathered}
\Phi_{1}=\left(\begin{array}{c}
\phi_{1}^{-} \\
\frac{1}{\sqrt{2}}\left(v_{1}+\phi_{1}^{0}+i \chi_{1}\right)
\end{array}\right), \Phi_{2}=e^{i \theta}\left(\begin{array}{c}
\phi_{2}^{+} \\
\frac{1}{\sqrt{2}}\left(v_{2}+\phi_{2}^{0}+i \chi_{2}\right)
\end{array}\right), \\
S=\frac{1}{\sqrt{2}} e^{i \varphi}\left(v_{3}+\phi_{3}^{0}+i \chi_{3}\right)
\end{gathered}
$$

We can see that vacuum expectation values (vevs) of Higgs fields are included into the neutralino mass matrix. In this case there are more possibilities for construct different scenarios of investigation. But if we will add mixing of CP-odd and CP-even Higgs bosons states the number of possibilities will decrease radically. This way $\mathrm{CP}$ violation was considered in the work [8]. In this case there is the problem to calculate the mass of the Higgs boson, therefore, it remains limited number of scenarios studies [23] in accordance with local minimum physical condition. Consequently, there are only two values for the particle candidate of dark matter. Also there are constrains on parameters from Electric Dipole Moments [24].

\section{Conclusion}

We have reviewed the existing limits on masses of dark matter particles in the various experimental data. It should be noted that supersymmetric particle neutralino is still relevant for the study. Moreover, non-minimal supersymmetric model can quite well describe the available results and predict new 
scenarios of research. However, it is necessary to consider changing the ranges of possible values of free parameters. So, we can conclude that the mass of the lightest neutralino does not exceed $1 \mathrm{TeV}$. In this case, the value $v_{3}$ has a strong influence on the mass range. As can be seen from the results of the scan region of NMSSM free parameters the mass of the neutralino reaches close to $1 \mathrm{TeV}$ at values of $v_{3}=1000 \mathrm{GeV}$. We also indicated that when considering CP-violation free parameters become larger, but this does not always lead to the valuable possibilities for theoretical predictions. In addition, there are some other ways to restrict values of the parameters, for example, the Electric Dipole Moments calculation.

\section{References}

[1] F. Zwicky, ApJ V.86, 217 (1937).

[2] D. N. Spergel, L. Verde, H. V. Peiris, E. Komatsu, M. R. Nolta, C. L. Bennett, M. Halpern, G. Hinshaw, N. Jarosik, A. Kogut, et al., ApJS 148, 175 (2003), astro-ph/0302209.

[3] D. S. Gorbunov, V. A. Rubakov, Introduction to the theory of the early Universe (Lenand, 2016).

[4] J.Rosiek, Physical Review D, V.41, 3464-3501 (1990).

[5] M.Maniatis, IJMP A., V.25, 3505-2602 (2010).

[6] B.A. Dobrescu, K.T. Matchev, JHEP, V.2000, (2000).

[7] D.J. Miller, R. Nevzorov, arXiv:hep-ph/0309143v1

[8] Gurskaya A.V., Dolgopolov M.V., Vestn. Samar. Gos. Tekhn. Univ. Ser. Fiz.-Mat. Nauki 20:4, 581-588 (2016).

[9] A. D. Sakharov, JETP Lett. 5, 24 (1967).

[10] https://twiki.cern.ch/twiki/bin/view/AtlasPublic/SupersymmetryPublicResults

[11] Mendez M.del R.A. et al., Journal of Physics:Conference Series, V.468, 012006 (2013).

[12] Lewin, J. D., Smith, P. F., Astropart. Phys. 6, 87-112 (1996).

[13] J.Liu, X.Chen, X.Ji, Nature Physics, 13, 212-216 (2017).

[14] Tan, A. et al. (PandaX-II Collaboration)Phys. Rev. Lett. 117, 121303 (2016).

[15] Akerib, D. S. et al. (LUX Collaboration) Phys. Rev. Lett. 118, 021303 (2017).

[16] Agnese, R. et al. (SuperCDMS Collaboration) Phys. Rev. Lett. 116, 071301 (2016).

[17] Angloher, G. et al. (CRESST Collaboration) Eur. Phys. J. C 76, 25 (2016).

[18] G.Bertone, D.Hooper, J.Silk, Phys. Rep. 405, 279 (2005).

[19] Chan, M.H. Astrophys Space Sci (2017) 362: 147.

[20] D.A.Vasquez, G.Belanger, C.Boehm, A.Pukhov, J.Silk Phys. Rev. D 82, 115027 (2010).

[21] M. M. Almarashi, S. Moretti Phys. Rev. D 83, 035023 (2011).

[22] Gurskaya A.V., Filippov Yu.P. Teoreticheskaya fizika [Theoretical Physics], 12, 69-81 (2013).

[23] Gurskaya A.V., Dolgopolov M.V., EPJ Web of Conferences, V.125, 02011 (2016)

[24] S.F.King, M.Muhlleitner, R.Nevzorov, K.Walz, Nuclear Physics B 901, 526-555 (2015). 